\title{
List of frequently met concepts and notations
}

\section{Set theory}

- $|M|$ is the cardinality of a set $M$ (if $G$ is a group, then $|G|$ is called the order of $G$ ).

- $x \in M$ means that $x$ is an element of $M . N \subseteq M$ means that $N$ is a subset of $M$; if $N \neq M$, we write $N \subset M$.

- $\emptyset$ is the empty set.

- $\quad N$ is called a nontrivial subset of $M$, if $N \neq \emptyset$ and $N \subseteq M$. If $N \subset M$, we say that $N$ is a proper subset of $M$.

- $M \cap N$ is the intersection and $M \cup N$ is the union of sets $M$ and $N$. If $M, N$ are sets, then $N-M$ is the difference of $N$ and $M$.

- $\quad \mathbb{C}$ is the set (field) of complex numbers.

- $\quad \mathbb{R}$ is the set (field) of real numbers.

- $\mathbb{Q}$ is the set (field) of rational numbers.

- $\mathbb{Z}$ is the set (ring) of integers: $\mathbb{Z}=\{0, \pm 1, \pm 2, \ldots\}$.

- $\mathbb{N}$ is the set of natural numbers.

\section{Number theory and general algebra}

- $\quad p$ is always a prime number.

- $\quad m, n$ are always natural numbers.

- $\operatorname{GCD}(m, n)$ is the greatest common divisor of $m$ and $n$.

- $\quad m \mid n$ should be read as follows: $m$ divides $n$.

- $\pi(m)$ is the set of all prime divisors of $m$.

- $\pi$ is a set of primes (it may be the empty set).

- $\pi^{\prime}$ is the set of primes not contained in $\pi$.

- $m_{\pi}$ is the number satisfying the following three conditions: $\pi\left(m_{\pi}\right) \subset \pi, m_{\pi} \mid m$, $\pi\left(m / m_{\pi}\right) \subset \pi^{\prime}$.

- We write $m_{p}, p^{\prime}$ instead of $m_{\{p\}},\left\{p^{\prime}\right\}$, respectively.

- $\quad m$ is a $\pi$-number if $\pi(m) \subseteq \pi$ (or $m_{\pi}=m$ ).

- $\mathrm{GF}\left(p^{m}\right)$ is the finite field containing $p^{m}$ elements.

- $\quad \mathbb{F}^{*}$ is the multiplicative group of a field $\mathbb{F}$.

- $\quad \mathbb{F}^{n}$ is the $n$-dimensional vector space over $\mathbb{F}$.

- $\quad \mathbb{F}_{n}$ is the set of all $n \times n$ matrices over $\mathbb{F}$.

- If $A$ is a square matrix, then $\operatorname{det} A$ and $\operatorname{tr} A$ are the determinant and the trace of $A$ (that is, the sum of elements on its principal diagonal), respectively.

- $I_{n}$ is the $n \times n$ identity matrix.

- $\bar{\alpha}$ is the number conjugate to $\alpha \in \mathbb{C}$.

- $\quad[x]$ is the integer part of $x \in \mathbb{R}$. 


\section{Groups}

- $G$ is always a finite group.

- $H \leq G$ means that $H$ is a subgroup of $G$.

- $H<G$ means that $H \subseteq G$ and $H \neq G$ (in this case $H$ is called a proper subgroup of $G$ ); $\{1\}$ denotes the group of order 1 ; $H$ is a nontrivial subgroup of $G$ if $\{1\}<H<G$.

- $H$ is a maximal subgroup of $G$ if $H<G$ and $H \leq M<G$ imply that $H=M$.

- $H \unlhd G$ means that $H$ is a normal subgroup of $G$; moreover, if $H \neq G$, we write $H \triangleleft G$ and say that $H$ is a proper normal subgroup of $G . H \triangleleft G$ is called a nontrivial normal subgroup of $G$ if $|H|>1$.

- $\quad H$ is a minimal normal subgroup of $G$ if (a) $H \unlhd G$, (b) $H>\{1\}$, (c) $N \triangleleft G$ and $N<H$ imply $N=\{e\}$. Thus, $\{1\}$ has no minimal normal subgroups.

- $G$ is simple if it is a minimal normal subgroup of $G$ (in particular, $|G|>1$ ).

- $\quad H$ is a maximal normal subgroup of $G$ if $G / H$ is simple.

- $G$ is a monolith if $G=\{1\}$ or if $G$ contains only one minimal normal subgroup.

- The subgroup generated by all minimal normal subgroups of $G$ is called the socle of $G$ and is denoted by $\operatorname{Sc}(G)$. One can represent $\operatorname{Sc}(G)$ as the direct product of certain minimal normal subgroups of $G$. We put $\operatorname{Sc}(\{1\})=\{1\}$. Obviously, $\operatorname{Sc}(G)$ is a characteristic subgroup of $G$.

- $\mathrm{N}_{G}(M)=\left\{x \in G \mid x^{-1} M x=M\right\}$ is the normalizer of a subset $M$ in $G$.

- $\mathrm{C}_{G}(x)$ is the centralizer of an element $x$ in $G: \mathrm{C}_{G}(x)=\{z \in G \mid z x=x z\}$.

- $\mathrm{C}_{G}(M)=\bigcap_{x \in M} \mathrm{C}_{G}(x)$ is the centralizer of a subset $M$ in $G$.

- Aut $G$ is the group of all automorphisms of $G$ (the automorphism group of $G$ ).

- Inn $G$ is the group of all inner automorphisms of $G$.

- $\quad$ Out $G=$ Aut $G / \operatorname{Inn} G$.

- $[x, y]=x^{-1} y^{-1} x y$ is the commutator of elements $x, y$ of $G$. If $M, N \subset G$, then $[M, N]=\langle[x, y] \mid x \in M, y \in N\rangle$. (However, we use $[M, N]=\{[x, y] \mid x \in M, y \in N\}$ in Chapter XI.)

- If $M \subset G$, then $\langle M\rangle$ is the subgroup of $G$ generated by $M$.

- $G^{\prime}$ is the subgroup generated by all commutators $[x, y], x, y \in G$ (i.e., $G^{\prime}=[G, G]$ ), $G^{\prime \prime}=\left(G^{\prime}\right)^{\prime}, G^{\prime \prime \prime}=\left(G^{\prime \prime}\right)^{\prime}$ and so on.

- $Z(G)=\bigcap_{x \in G} C_{G}(x)$ is the center of $G$.

- $\Phi(G)$ is the Frattini subgroup of $G$ (the intersection of all maximal subgroups of $G$ ).

- $\mathrm{F}(G)$ is the Fitting subgroup of $G$ (the maximal normal nilpotent subgroup of $G$ ).

- $\mathrm{S}(G)$ is the solvable radical of $G$ (the maximal solvable normal subgroup of $G$ ).

- $\quad \exp G$ is the exponent of $G$ (the least common multiple of the orders of the elements of $G)$.

- $\mathrm{o}(x)$ is the order of an element $x$ of $G$.

- $\mathrm{k}(G)$ is the number of conjugacy classes of $G$ (= $G$-classes), the class number of $G$.

- If $M \subseteq G$, then $\mathrm{k}_{G}(M)$ is the number of $G$-classes containing elements of $M$.

- $\pi(G)=\pi(|G|)$. 
- $\mathrm{O}_{\pi}(G)$ is the maximal normal $\pi$-subgroup of $G, \mathrm{O}(G)=\mathrm{O}_{2^{\prime}}(G)$ (obviously, one has $\left.\mathrm{O}_{p}(G) \in \operatorname{Syl}_{p}(\mathrm{~F}(G))\right)$.

- $\mathrm{O}^{\pi}(G)$ is the subgroup generated by all $\pi^{\prime}$-elements of $G$.

- $\quad \mathrm{C}_{m}$ is the cyclic group of order $m$.

- $A \times B$ is the direct product of groups $A$ and $B$.

- $\quad A * B$ is a central product of groups $A$ and $B$.

- $G^{0}=\{1\} ; G^{m}$ is the direct product of $m$ copies of $G$.

- $\quad \mathrm{E}_{p^{m}}=\left(\mathrm{C}_{p}\right)^{m}$ is the elementary abelian group of order $p^{m}$.

- A group $G$ is said to be homocyclic if it is a direct product of isomorphic cyclic subgroups (obviously, elementary abelian $p$-groups are homocyclic ).

- $\quad \mathrm{ES}(m, p)$ is an extraspecial group of order $p^{l+2 m}$ (a $p$-group $G$ is said to be extraspecial if $G^{\prime}=\Phi(G)=Z(G)$ is of order $p$ ).

- A special $p$-group is a nonabelian $p$-group $G$ such that $G^{\prime}=\Phi(G)=Z(G)$ is elementary abelian.

- $\quad(A, B)$ is a Frobenius group with kernel $B$ and Frobenius complement $A$ ( $A$ and $B$ do not determine $(A, B)$ up to isomorphism).

- $\quad \mathrm{D}_{2 m}$ is the dihedral group of order $2 m, m>2$.

- $\quad \mathrm{Q}_{2^{m}}$ is the generalized quaternion group of order $2^{m}>2, m>3$.

- $\quad \mathrm{SD}_{2^{m}}$ is the semidihedral group of order $2^{m} \geq 2^{4}$.

- $\quad \mathrm{cl} G$ is the nilpotency class of a $p$-group $G$.

- $\quad C L G$ is the set of all $G$-classes.

- A $p$-group of maximal class is a nonabelian group $G$ of order $p^{m}$ with $\operatorname{cl} G=m-1$.

- If $G$ is a $p$-group, then $\Omega_{m}(G)=\left\langle x \in G \mid x^{p^{m}}=1\right\rangle$, and $m \cdot G=\left\langle x^{m} \mid x \in G\right\rangle$.

- $\operatorname{Syl}(G)$ is the set of all Sylow subgroups of $G$.

- $\operatorname{Syl}_{p}(G)$ is the set of all Sylow $p$-subgroups of $G$.

- $\quad H$ is a Hall subgroup of $G$ if $(|H|,|G: H|)=1$.

- $\quad H$ is a $\pi$-Hall subgroup of $G$ if $|H|=|G|_{\pi}$.

- $\mathrm{S}_{n}$ is the symmetric group of degree $n$.

- $\quad \mathrm{A}_{n}$ is the alternating group of degree $n$.

- $\mathrm{GL}(n, F)$ is the set of all nonsingular $n \times n$ matrices with entries in a field $F$, the general linear group over $F$.

- $\operatorname{SL}(n, F)=\{A \in \mathrm{GL}(n, F) \mid \operatorname{det} A=1 \in F\}$, the special linear group over $F$.

- $\quad \operatorname{PGL}(m, F)=\mathrm{GL}(n, F) / \mathrm{Z}(\mathrm{GL}(n, F))$.

- $\operatorname{PSL}(n, F)=\operatorname{SL}(n, F) / Z(\operatorname{SL}(n, F))$.

- $\operatorname{AGL}(n, F)$ is the natural extension of $F^{n}$ by GL $(n, F)$, the affine general linear group.

- $\quad \mathrm{Sz}\left(2^{m}\right)$ is the simple Suzuki group, $m>1$ being odd.

- For $H<G, H_{G}=\bigcap_{x \in G} x^{-1} H x$ is called the core of the subgroup $H$ in $G$. Obviously, $H_{G} \unlhd G$.

- An element $x \in G$ is a $\pi$-element if $\pi(\mathrm{o}(x)) \subseteq \pi$.

- $G$ is a $\pi$-group if $\pi(G) \subseteq \pi$. Obviously, $G$ is a $\pi$-group if and only if all its elements are $\pi$-elements.

- $\mathrm{O}^{\pi}(G)=\left\langle x \in G \mid \pi(\mathrm{o}(x)) \subseteq \pi^{\prime}\right\rangle$. 
- $\mathrm{O}^{\pi, \sigma}(G)=\mathrm{O}^{\sigma}\left(\mathrm{O}^{\pi}(G)\right)$.

- A group $G$ is an extension of $N \unlhd G$ by a group $H$ if $G / N \simeq H$. A group $G$ splits over $N$ if $G=H N$ with $H<G$ and $H \cap N=1$ (in that case, $G$ is a semidirect product of $H$ and $N$ with kernel $N$ ).

- A group $G$ is $p$-solvable if all indices of its composition series are equal to $p$ or are $p^{\prime}$-numbers. A group $G$ is $\pi$-solvable if it is $p$-solvable for all $p \in \pi$.

- A group $G$ is said to be $\pi$-separable if all indices of its composition series are $\pi$ - or $\pi^{\prime}$-numbers.

- If $M \subseteq G, x \in G$, then $M^{x}=x^{-1} M x=\left\{x^{-1} a x \mid a \in M\right\}$.

- $\quad H$ is a TI-subgroup of $G$ if $H \cap H^{x}=1$ for all $x \in G-\mathrm{N}_{G}(H)$; $M$ is a TI-subset of $G$ if $M \cap M^{x} \subseteq\{1\}$ for all $x \in G-\mathrm{N}_{G}(M)$.

- $H^{\#}=H-\left\{e_{H}\right\}$, where $e_{H}$ is the identity element of the group $H$. If $M \subseteq G$, then $M^{\#}=M-\left\{e_{G}\right\}$.

- A permutation $\sigma$ of a set $M$ is regular if $\sigma(x) \neq x$ for all $x \in M$. An automorphism $a$ of $G$ is regular (= fixed-point free) if it induces a regular permutation on $G^{\#}$.

- If $x, y \in G$, then the expression " $x \sim y$ in $G$ ” means that $x, y$ are conjugate in $G$. Similarly, " $M \sim N$ in $G$ " means that the subsets $M, N$ are conjugate in $G$.

- An involution is an element of order 2 in a group.

- An element $x \in G$ is real if $x \sim x^{-1}$ in $G$. An element $x$ is rational if all generators of the subgroup $\langle x\rangle$ are conjugate in $G$. An involution is a real and rational element.

- A section of a group $G$ is an epimorphic image of some subgroup of $G$.

- A group $G$ is $p$-closed if $\left|\operatorname{Syl}_{p}(G)\right|=1$ (i.e., $O_{p}(G) \in \operatorname{Syl}_{p}(G)$ ).

- A group $G$ is $p$-nilpotent if it has a normal $p$-complement, i.e., a normal subgroup $H$ of order $|G|_{p^{\prime}}$.

- $\quad \operatorname{An~} \mathrm{S}\left(p^{a}, q^{b}, q^{c}\right)$-group is a $q$-closed minimal nonnilpotent group $G$ of order $p^{a} q^{b+c}$ with $|\mathrm{Z}(G)|=p^{a-1} q^{c}$ (see Chapter XI).

- If $F=\mathrm{GF}\left(p^{n}\right)$, then we write $\mathrm{GL}\left(m, p^{n}\right)$, etc., instead of $\mathrm{GL}(m, F)$, etc.

- If $M \subseteq G$, then $M^{G}$ is the normal closure of $M$ in $G$.

\section{Characters and representations}

- $\mathrm{F}[G]$ is the set of all functions from $G$ to $\mathbb{C}$.

- $\quad \mathrm{CF}[G]$ is the set of all central (= class) functions from $G$ to $\mathbb{C}$.

- $\operatorname{Char}(G)$ is the set of all complex characters of $G$. It is convenient to consider the zero function $\mathrm{O}_{G \rightarrow \mathbb{C}}$ as an element of the set $\operatorname{Char}(G)$.

- $\operatorname{Irr}(G)$ is the set of all irreducible characters of $G$.

- A character of degree 1 is said to be linear.

- $\operatorname{Lin}(G)$ is the set of all linear characters of $G$ (obviously, $\operatorname{Irr}(G) \subseteq \operatorname{Irr}(G)$ ).

- $\operatorname{Irr}_{1}(G)=\operatorname{Irr}(G)-\operatorname{Lin}(G)$ is the set of all nonlinear irreducible characters of $G$; $\mathrm{n}(G)=\left|\operatorname{Irr}_{1}(G)\right|$ is the number of nonlinear irreducible characters of $G$. 
- A class function $\theta$ is said to be a generalized character of $G$ if $\theta=\chi_{1}-\chi_{2}$, where $\chi_{1}, \chi_{2} \in \operatorname{Char}(G)$.

- $\operatorname{Ch}(G)$ is the set of all generalized characters of $G$.

- If $\theta, \lambda \in F[G], x \in G$, then $(\theta \lambda)(x)=\theta(x) \lambda(x)$.

- $\quad F G$ is the group algebra of $G$ over the field $F$.

- $\chi(1)$ is the degree of a character $\chi$ of $G$; $\operatorname{deg} T$ is the degree of a representation $T$ of $G$.

- If $\chi \in \operatorname{Char}(G), \phi \in \operatorname{Char}(H), H<G$, then $\chi_{H}$ is the restriction of $\chi$ to $H$, and $\phi^{G}$ is the induced character $\left(\phi^{G} \in \operatorname{Char}(G)\right)$.

- If $\vartheta, \psi \in \mathrm{CF}[G]$, then $\langle\vartheta, \psi\rangle=|G|^{-1} \sum_{x \in G} \vartheta(x) \overline{\psi(x)}$ is the scalar (or inner) product of $\vartheta$ and $\psi$.

- If $H \leq G, \phi \in \operatorname{Irr}(H)$, then $I_{G}(\phi)=\left\{x \in G \mid \phi^{x}=\phi\right\}$ is the inertia group of $\phi$ in $G$ (where $\phi^{x}(h)=\phi\left(x h x^{-1}\right)$ for $h \in H$ ).

- If $H \leq G$ and $\phi \in \mathrm{CF}[H]$, then $\dot{\phi}$ is the function in $\mathrm{CF}[G]$ that coincides with $\phi$ on $H$ and vanishes on $G-H$.

- $1_{G}$ is the principal character of $G\left(1_{G}(x)=1\right.$ for all $\left.x \in G\right)$.

- $\rho_{G}$ is the regular character of $G$.

- $\operatorname{Irr}(\chi)$ is the set of all irreducible constituents of a character $\chi$ of $G$. Furthermore, $\operatorname{Irr}_{1}(\chi)=\operatorname{Irr}(\chi) \cap \operatorname{Irr}_{1}(G)$. (The expression $\psi \in \operatorname{Irr}(\chi)$ means that the character $\psi$ is a constituent of $\chi$.)

- $\mathrm{X}(G)$ is the character table of $G$, and $\mathrm{X}_{1}(G)$ is its first column (consisting of the degrees of irreducible characters, counting multiplicities).

- $\mathrm{M}(G)$ is the Schur multiplier of $G$.

- If $M$ is a set, the Kronecker symbol $\delta: M \times M \rightarrow\{0,1\}$ is defined as follows: if $a=b$, then $\delta_{a, b}=1$, and if $a \neq b$, then $\delta_{a, b}=0$.

- $\quad \operatorname{cd} G=\{\chi(1) \mid \chi \in \operatorname{Irr}(G)\}$.

- $\operatorname{cd}_{1} G=\left\{\chi(1) \mid \chi \in \operatorname{Irr}_{1}(G)\right\}=\operatorname{cd} G-\{1\}$.

- $\mathrm{b}(G)=\max \{n \mid n \in \operatorname{cd} G\}$.

- $\quad \operatorname{ker} T$ is the kernel of a representation $T$.

- $\operatorname{ker} \chi$ is the kernel of a character $\chi$.

- $\mathrm{Z}(\chi)=\{\chi \in G|| \chi(\chi) \mid=\chi(1)\}$ is the quasikernel of $\chi \in \operatorname{Char}(G)$.

- $\mathrm{T}_{\chi}=\{x \in G \mid \chi(x)=0\}$ is the set of zeros of $\chi \in \operatorname{Ch}(G)$.

- $\mathrm{U}_{\chi}=\{\chi \in G|| \chi(x) \mid=1\}$ is the set of $\chi$-unitary elements of $G$ (where $\chi \in \operatorname{Ch}(G)$ ).

- Let $N \unlhd G$. Then $\operatorname{Irr}_{N}(G)=\{\chi \in \operatorname{Irr}(G) \mid N<\operatorname{ker} \chi\}$. We often identify the $\operatorname{sets} \operatorname{Irr}_{N}(G)$ and $\operatorname{Irr}(G / N)$. Next, $\operatorname{Irr}(G, N)=\operatorname{Irr}(G)-\operatorname{Irr}(G / N) ; \operatorname{Lin}_{N}(G)=\operatorname{Lin}(G) \cap \operatorname{Irr}_{N}(G)$.

- $\operatorname{Irr}_{\phi}(G)=\left\{\chi \in \operatorname{Irr}(G) \mid\left\langle\chi_{N}, \phi\right\rangle>0\right\}$, where $N \unlhd G, \phi \in \operatorname{Irr}(N)$.

- Let $H<G, \phi \in \operatorname{Irr}(H), \chi \in \operatorname{Irr}(G)$. Then $\chi$ is an extension of $\phi$ to $G$ if $\chi_{H}=\phi$.

- $v_{2}(\chi)$ is the Frobenius-Schur indicator of $\chi \in \operatorname{Irr}(G)$ (see Chapter IV).

- $\operatorname{mc}(G)=\mathrm{k}(G) /|G|$ is the measure of commutativity of $G$.

- $\mathrm{T}(G)=\sum_{\chi \in \operatorname{Irr}(G)} \chi(1)$, and $f(G)=\mathrm{T}(G) /|G|$.

- Let $T$ be a representation, affording the character $\chi$ of $G$. Then the function $\operatorname{det} \chi: G \rightarrow \mathbb{C}^{*}$ is defined by $\operatorname{det}(\chi)(\chi)=\operatorname{det} T(x), \chi \in G$. Obviously, $\operatorname{det} \chi \in \operatorname{Lin}(G)$. 
- If $\chi \in \operatorname{CF}(G)$, then $\bar{\chi}: G \rightarrow \mathbb{C}$ is defined by $\bar{\chi}(x)=\overline{\chi(x)}, x \in G$.

- If $X \subseteq \operatorname{Irr}(G)$, then $X^{\#}=X-1_{G}$. In particular, $\operatorname{Irr}^{\#}(G)$ is the set of all nonprincipal characters of $G$. $\operatorname{Irr}_{1}\left(G, p^{\prime}\right)=\left\{\chi \in \operatorname{Irr}_{1}(G) \mid p+\chi(1)\right\}$.

- $\quad \mathrm{T}_{1}\left(G, p^{\prime}\right)=\sum_{\chi \in \operatorname{Irr}_{1}\left(G, p^{\prime}\right)} \chi(1)$.

- If $P \in \operatorname{Syl}_{p}(G)$, then $\mathrm{T}_{1}\left(G, P, p^{\prime}\right)=\sum_{\chi \in \operatorname{Irr}_{1}\left(G, p^{\prime}\right) P \notin \operatorname{ker} \chi} \chi(1)$.

- $\quad \operatorname{Kern} G=\left\{\operatorname{ker} \chi \mid \chi \in \operatorname{Irr}_{1}(G)\right\}$.

- $\quad v(x)=|\{\chi(x) \mid x \in G\}|$.

- A character $\chi$ of $G$ is monolithic if $\chi \in \operatorname{Irr}(G)$ and $G / \operatorname{ker} \chi$ is a monolith. $\operatorname{Irr}_{m}(G)$ is the set of all monolithic characters of $G, \operatorname{Irr}_{1, m}(G)=\operatorname{Irr}_{m}(G) \cap \operatorname{Irr}_{1}(G)$. 